\title{
Relação Conjugal no Contexto de Reprodução Assistida: O Tratamento e a Gravidez ${ }^{1}$
}

\author{
Isabela Machado Silva ${ }^{2}$ \\ Rita de Cássia Sobreira Lopes \\ Universidade Federal do Rio Grande do Sul
}

\begin{abstract}
RESUMO - Neste estudo de caso coletivo, investigou-se a relação conjugal de três casais que conceberam com o auxílio das Técnicas de Reprodução Assistida (TRA). Os dados, obtidos a partir de entrevistas individuais semiestruturadas, foram submetidos à análise de conteúdo qualitativa, embasada em três categorias: coesão, sexualidade e comunicação, durante o tratamento e a gravidez. O tratamento mostrou-se fonte potencial de dificuldades nas três dimensões. Durante a gravidez, permaneceram algumas dificuldades, especialmente na sexualidade, mas relataram-se aumento na coesão e redução de conflitos. Identificou-se a interação das três dimensões, de forma que uma pode tanto compensar dificuldades nas demais como contribuir para o funcionamento destas. Destaca-se, assim, a importância de considerar as diferentes dimensões da relação conjugal, em estudos com casais.
\end{abstract}

Palavras-chave: Gravidez, reprodução assistida, relação conjugal

\section{Marital Relationship in the Context of Assisted Reproduction: Treatment and Pregnancy}

\begin{abstract}
This collective-case study investigated marital relationship during pregnancy, in three couples who conceived through assisted reproduction techniques. The data, derived from individual semi-structured interviews, were submitted to a qualitative content analysis, based on three categories: cohesion, sexuality and communication, during treatment and pregnancy. The treatment was a source of potential difficulties in the three categories. During pregnancy, some difficulties remained, especially in couples' sexuality, but there was an increase in cohesion and a reduction in conflicts. An interaction of the three dimensions was identified, so that one dimension could either compensate difficulties in the other dimensions or contribute to their functioning. The importance of investigating different dimensions of the marital relationship in studies with couples is highlighted.
\end{abstract}

Keywords: Pregnancy, assisted reproduction, marital relations

A abordagem desenvolvimental da família a compreende como um sistema que se modifica no decorrer do tempo para lidar com os diversos eventos com que se depara (Andolfi, Ângelo, Menghi, \& Nicolo-Gorigliano, 1989; Carter \& McGoldrick, 1995; Glick \& Kessler, 1980). Dessa forma, assim como os indivíduos, as famílias (Carter \& McGoldrick, 1995; Cerveny, 2002) e os casais (Pittman, 1987) também apresentam um ciclo vital, ao longo do qual vivenciam alterações nas relações entre seus membros. Conforme assinalaram Lavee, McCubbin e Olson (1987), essas mudanças podem ser originadas tanto por transições esperadas entre os diferentes estágios do ciclo vital - como a transição para a parentalidade -, como por eventos inesperados com que a família se depara ao longo da sua trajetória - como a constatação de uma situação de infertilidade. No entanto, de forma geral, esses momentos de transformação tendem a ser geradores de estresse para o sistema (Boss, 1980; Carter \& McGoldrick, 1995).

1 Trabalho derivado da dissertação de mestrado da primeira autora, orientada pela segunda. Apoio: CNPq e FAPERGS.

2 Endereço para correspondência: Rua Ramiro Barcelos, 2600, sala 108, Santa Cecília. Porto Alegre, RS. CEP 90035-003.E-mail: isabela.ms@ gmail.com
Embora toda família experiencie estressores ao longo do seu ciclo vital, a forma como cada uma reage frente aos mesmos e seus efeitos a longo prazo são variados, sendo que se pode encontrar tanto o crescimento familiar como o surgimento de dificuldades (Boss, 1980). Diversos elementos podem influenciar a adaptação da família frente a um evento estressor (Pittman, 1987). A partir de uma revisão da literatura, Olson (1991) concluiu que três dimensões principais devem ser levadas em consideração no estudo da dinâmica familiar e conjugal: a coesão, que se refere ao grau de ligação entre os membros; a adaptabilidade, que remete à capacidade de adaptação do sistema frente a mudanças e a comunicação, que interfere nas outras dimensões.

A transição para a parentalidade é considerada como uma das transições que gera maior estresse na vida do casal (Pittman, 1987). O surgimento de novas demandas e prioridades exige dos cônjuges uma reelaboração da imagem que fazem de si mesmos, do outro e do próprio relacionamento conjugal, o que leva a sentimentos ambivalentes e a mudanças na forma de se relacionar adotada até então (Berthoud, 2002; Stern, 1997). A alteração da configuração familiar advinda da entrada de um novo membro pode vir, ainda, a afetar dimensões importantes da relação conjugal, como a intimidade, a comunicação e o sexo (Bradt, 1995), sendo que 
a gravidez é considerada justamente como o período em que os casais se preparam física e psicologicamente para todas essas mudanças (Brazelton \& Cramer, 1992).

Já a infertilidade representa, segundo Burns (1987), justamente um obstáculo no caminho rumo à parentalidade, sendo considerada, assim, um evento estressor. Diante da constatação de uma situação de infertilidade, tornam-se necessárias diversas decisões e redefinições, que podem envolver expectativas, metas, relacionamentos e, até mesmo, a própria identidade dos cônjuges (Burns, 1987; Leiblum, 1997; McDaniel, Hepworth, \& Doherty, 1994; Sharf \& Weinshel, 2002). Quando o casal opta pela realização das técnicas de reprodução assistida (TRA), os cônjuges se deparam com uma série de questões com as quais necessitarão lidar, como, por exemplo, a adequação da rotina - inclusive a sexual - aos procedimentos, o contato frequente com a equipe médica, possíveis frustrações quanto aos resultados do tratamento e a decisão sobre com quem compartilhar essas experiências (Burns, 1987; Leiblum, 1997). Nesse contexto, o casal pode se sentir excluído por considerar-se diferente da maioria e vivenciar sentimentos de raiva, culpa e vergonha, o que tende a repercutir nas diferentes relações desses indivíduos, especialmente a conjugal (Burns 1987; Leiblum, 1997; Schaffer \& Diamond, 1994; Seger-Jacob, 2006; Shapiro, 1992).

Dessa forma, ao pensarmos em casais que engravidam com o auxílio das TRA, devemos considerar que os mesmos vivenciam dois estressores consecutivos: a experiência da infertilidade e a posterior transição para a parentalidade, $\mathrm{o}$ que pode influenciar a vivência deste momento e os futuros relacionamentos familiares (Hjelmstedt, Widstrom \& Collins, 2006). Alguns autores (Ulrich, Gagel, Hemmerling, Pastor \& Kentenich, 2004) sugeriram, inclusive, que, ao atravessarem a transição para a parentalidade, esses casais vivenciam uma dupla tarefa desenvolvimental, pois, ao mesmo tempo em que precisam fazer os ajustes para a chegada do bebê, também experienciam mudanças em sua própria identidade decorrentes da superação da infertilidade.

Pesquisas foram desenvolvidas com o intuito de verificar como casais que engravidaram a partir das TRA vivenciam o período da gestação (Cohen, McMahon, Tennant, Saunders, \& Leslie, 2000; Fisher, Hammarberg, \& Baker, 2008; Kloch \& Greenfeld, 2000; Stanton \& Golombok, 1993; Sydsjö, Wadsby, Kjellberg, \& Sydsjö, 2002; Ulrich et al., 2004). Conforme Ulrich et al. (2004), os resultados obtidos por esses estudos sugerem que existem mais semelhanças do que diferenças entre casais que recorreram às TRA e aqueles que conceberam naturalmente. No entanto, alguns autores constataram certas especificidades, sendo possível perceber divergências entre os estudos. Por um lado, identificaram-se, nos casais que engravidaram por reprodução assistida, uma avaliação mais positiva da relação no que se refere à satisfação conjugal, à comunicação e à resolução de conflitos (Sydsjö et al., 2002), assim como a descrição, segundo as esposas, de um relacionamento mais afetuoso, sensível e companheiro e menos coercitivo, crítico e dominador (Fisher et al., 2008). Por outro lado, alguns estudos relataram um pior ajustamento conjugal e a percepção de um relacionamento menos cuidadoso entre os cônjuges, de acordo com a perspectiva dos maridos (Cohen et al., 2000), uma menor preocupação, por parte das esposas, com a relação conjugal (Kloch \& Greenfeld, 2000) e maiores dificuldades sexuais (Ulrich et al., 2004).

No entanto, em uma revisão recente desses estudos (Silva \& Lopes, 2009), constatou-se o predomínio do uso de escalas e de análises quantitativas para a avaliação de variáveis específicas, tais como satisfação e ajustamento conjugal. Percebe-se, portanto, a falta de estudos que busquem descrever a experiência desses casais. Dada a complexidade dos fatores envolvidos, acredita-se que um estudo de caso qualitativo poderá servir a esse fim, contribuindo para a compreensão mais detalhada e contextualizada do tema (Robson, 1993).

Assim, o presente estudo teve como objetivo investigar a relação conjugal durante o tratamento e a gravidez, em casais que engravidaram com o auxílio das TRA. A partir da sugestão de Olson (1991) sobre quais dimensões devem ser priorizadas na investigação da dinâmica familiar e conjugal, assim como do levantamento dos aspectos que, segundo a literatura, seriam influenciados pela experiência da infertilidade e da parentalidade, selecionaram-se três dimensões a serem investigadas neste trabalho: coesão, sexualidade e comunicação.

\section{Método}

\section{Participantes}

Os participantes deste estudo fazem parte de um projeto maior, intitulado 'Transição para a parentalidade e a relação conjugal no contexto da reprodução assistida: Da gestação ao primeiro ano de vida do bebê' (Lopes, Piccinini, Dornelles, Silva, \& Passos, 2007). Participam desse projeto casais que conceberam com o auxílio das TRA e que são indicados pelos profissionais que trabalham no Serviço de Ginecologia e Obstetrícia do Hospital de Clínicas de Porto Alegre (HCPA). Os participantes desse projeto são contatados em três momentos distintos: (a) no terceiro trimestre de gestação; (b) após o bebê completar três meses e (c) após o bebê completar um ano. Os dados apresentados neste trabalho referem-se apenas a entrevistas realizadas na primeira fase do projeto.

A partir dessa amostra mais ampla, selecionaram-se, com base no conceito de heterogeneidade (Patton, 2002), três casais que se encontravam no terceiro trimestre da gestação do primeiro filho do casal. Segundo Patton, a utilização desse critério, que se caracteriza pela variedade dos casos, permite que se identifiquem os temas centrais referentes ao contexto estudado, uma vez que se constatam tendências compartilhadas por casos que apresentam características diversas. Conforme poderá ser observado a seguir, os casais variaram quanto (a) à causa da infertilidade, aspecto que, segundo Lee, Sun e Chao (2001), pode influenciar o impacto da infertilidade sobre a relação conjugal; (b) a técnica utilizada, visto que cada técnica apresenta suas especificidades (Nichols \& Pace-Nichols, 2000); e (c) a existência de filhos de relações anteriores, visto que se acredita que a experiência de transição para a parentalidade tende a ser diferente para casais que já tiveram filhos e aqueles que esperam seu primeiro (Pereira \& Piccinini, 2007). 
Casal 01: Bianca e Eduardo ${ }^{3}$ iniciaram sua relação há aproximadamente dez anos. Ambos se encontravam na faixa dos 30 anos, completaram o ensino superior e trabalhavam. Seu nível socioeconômico era considerado médio ${ }^{4}$. A causa diagnosticada para a infertilidade foi considerada masculina. O casal se submeteu a um procedimento de fertilização in vitro. A gravidez foi alcançada na primeira tentativa com as TRA. Nenhum dos cônjuges possuía filhos de uniões anteriores.

Casal 02: Sílvia e Ricardo iniciaram sua relação há aproximadamente 15 anos. Ambos se encontravam na faixa dos 40 anos, completaram o ensino superior e trabalhavam. Seu nível socioeconômico era considerado médio-alto. A causa diagnosticada para a infertilidade foi considerada feminina. $\mathrm{O}$ casal se submeteu a um procedimento de doação de gametas. A gravidez foi alcançada na segunda tentativa com as TRA. Nenhum dos cônjuges possuía filhos de uniões anteriores.

Casal 03: Viviana e Daniel iniciaram sua relação há aproximadamente cinco anos. Ela estava na faixa dos 30 anos e ele, na dos 20. Ambos completaram o Ensino Fundamental, ele trabalhava e ela não exercia atividade remunerada. Seu nível socioeconômico era considerado médio-baixo. A causa diagnosticada para a infertilidade foi considerada feminina. $\mathrm{O}$ casal se submeteu a um procedimento de fertilização in vitro. A gravidez foi alcançada na segunda tentativa com as TRA. Um dos cônjuges possuía filhos de uniões anteriores.

\section{Delineamento e Procedimentos}

O presente trabalho se caracteriza como um estudo de caso coletivo (Stake, 1995). Examinaram-se tanto as particularidades de cada caso, como as semelhanças existentes entre eles, especificamente no que tange às três dimensões da relação conjugal investigadas: coesão, sexualidade e comunicação.

Após a indicação dos potenciais participantes pela equipe do HCPA, os mesmos eram contatados por telefone, momento em que o estudo lhes era apresentado e eles eram convidados a participar. Aproximadamente $26 \%$ dos potenciais participantes contatados por nossa equipe recusaram-se a colaborar. Entre os motivos alegados, mencionaram-se falta de interesse, pouca disponibilidade de tempo e desejo de "deixar para trás" essa experiência tão desgastante. Com aqueles que demonstraram interesse em participar, foi marcado um encontro, em que se apresentou o Termo de Consentimento Livre e Esclarecido (TCLE). Todos os casais concordaram com os termos propostos no TCLE, de forma que, nessa mesma oportunidade, teve início a realização da coleta de dados. Os membros do casal preencheram uma ficha de dados sociodemográficos e responderam individualmente a duas entrevistas semiestruturadas e a uma aplicação resumida do Teste de Apercepção Temática - TAT (Murray, 1943/2005), cujos dados não serão utilizados no presente trabalho. De for-

3 Para preservar a privacidade dos participantes, todos os nomes apresentados, neste trabalho, são fictícios.

4 O nível socioeconômico foi avaliado a partir da adaptação das categorias de Hollingshead proposta por Tudge e Frizzo (2002). Nessa escala, os casais se enquadraram respectivamente nas categorias 4,5 e 3 . ma geral, as entrevistas e a aplicação do teste foram realizadas simultaneamente com os dois cônjuges, por pesquisadoras diferentes. A aplicação dos instrumentos durava aproximadamente duas horas, sendo que, dependendo da disponibilidade do casal, poderia ser realizada em mais de um encontro. Os encontros se deram na própria residência dos participantes ou em seu local de trabalho, considerando o que lhes fosse mais conveniente. As entrevistas, assim como o TAT, foram gravadas em áudio e posteriormente transcritas para análise.

O projeto do qual o presente trabalho faz parte segue as diretrizes definidas na resolução da Comissão Nacional de Pesquisa (Conselho Nacional de Saúde, 1996). O referido projeto foi aprovado pelo Comitê de Ética em Pesquisa do HCPA. Garantiu-se que os participantes poderiam deixar de participar do estudo a qualquer momento, sem que isso lhes acarretasse quaisquer prejuízos, e que, em caso de desconforto durante a realização das entrevistas, os mesmos poderiam ser encaminhados a atendimento psicológico se assim o desejassem.

\section{Instrumentos}

Entre os instrumentos utilizados no projeto e cujos dados serão utilizados no presente trabalho, encontram-se uma ficha de dados sociodemográficos, que visa à obtenção de informações necessárias ao contato com os participantes e à sua caracterização, e duas entrevistas individuais semiestruturadas:

Entrevista sobre a gestação e as expectativas da gestante (NUDIF, 1998a) e entrevista sobre a gestação e as expectativas do futuro pai (NUDIF, 1998b): Compostas por diversos blocos de questões. Os primeiros abrangem percepções e sentimentos do participante quanto à gestação e ao feto. Os blocos seguintes abordam a relação com o cônjuge e com outros familiares, as expectativas sobre diferentes aspectos da vida familiar e a percepção do impacto dessas técnicas sobre a vivência da gestação.

Entrevista sobre a relação conjugal na gestação (Lopes, Silva, Dornelles, \& Piccinini, 2007, adaptado de Lopes \& Menezes, 2003). A primeira parte da entrevista refere-se à história da relação, incluindo questões relativas ao início do relacionamento e ao seu desenvolvimento posterior. A segunda parte é composta por questões que enfocam percepções e sentimentos quanto ao surgimento, no casal, da ideia de ter um filho, a decisão pela utilização das TRA e suas repercussões. A terceira parte abrange questões voltadas à relação no presente, enfocando a imagem do cônjuge, os pontos positivos e negativos da relação, a comunicação do casal, a vida sexual, as manifestações de afeto, os ideais de relacionamento, as relações com as redes de apoio e as atividades às quais os cônjuges costumam se dedicar. A quarta parte investiga a visão da família ampliada, enquanto a parte final volta-se às expectativas em relação ao futuro da relação.

\section{Análise dos dados}

Os dados foram submetidos à análise de conteúdo qualitativa, baseadas em categorias definidas a priori (Laville \& 
Dione, 1999). Inicialmente, as autoras realizaram uma primeira leitura do material com o intuito de se familiarizarem com seu conteúdo e de verificarem a adequação das categorias propostas para a análise. Tendo-se considerado as categorias adequadas, sua definição operacional foi discutida entre as autoras para se dirimirem possíveis dúvidas. As entrevistas foram, então, novamente lidas pela primeira autora deste estudo, que buscou identificar trechos pertinentes a cada uma das categorias, que se referem a diferentes dimensões da relação conjugal. Questões acerca da categorização dos conteúdos expressos pelos participantes foram resolvidas em conjunto pelas autoras. Assim, definiram-se como categorias: (1) Coesão (Olson, 1991; Rios-González, 2005): Descreve os conteúdos que retratam os sentimentos que unem os cônjuges, sua percepção de proximidade e o apoio entre ambos. (2) Sexualidade (Miller, Caughlin, \& Huston, 2003; Wright, 1998): Descreve os conteúdos referentes à vida sexual do casal e às suas manifestações físicas de afeto. (3) Comunicação (Olson, 2000; Snyder, Cozzi, \& Mangrum, 2006): Descreve o padrão de comunicação descrito pelos cônjuges, a expressão e a compreensão de sentimentos e ideias, os assuntos compartilhados pelo casal, os temas que levam a conflitos entre os cônjuges e a forma como eles são resolvidos.

A análise dos dados teve como objetivo descrever essas diferentes dimensões da relação conjugal, em dois momentos: durante o tratamento (investigado retrospectivamente) e a gravidez. A seguir, serão apresentados os dados referentes a cada uma dessas dimensões em ambos os momentos.

\section{Resultados e Discussão}

\section{Coesão: Durante o tratamento}

No que se refere à vivência dessa dimensão durante a realização do tratamento nos três casais observou-se a participação e o apoio dos maridos. Segundo Viviana (casal 03), o marido "participou desde o inicio mesmo. Sempre ali junto. Quando tinha que ir ao hospital, ele deixava de trabalhar e ia, nunca reclamou (...) ele me incentivava e me encorajava". Isso se mostra de acordo com os resultados obtidos por Sabatelli, Meth e Gavazzi (1988), segundo os quais as esposas tenderam a perceber um aumento no apoio emocional recebido de seus companheiros em função da experiência da infertilidade. A percepção desse apoio não parece estar necessariamente atrelada à participação do marido no tratamento, pois, segundo Spotorno, Silva e Lopes (2008), mesmo quando se queixam da ausência dos maridos nesses momentos, as esposas referem que eles se mostram mais atenciosos.

Por outro lado, também se identificou, no relato dos três casais, a ideia de que a experiência do tratamento e, em especial, o seu fracasso poderiam constituir uma ameaça ao vínculo conjugal e, consequentemente, à coesão do casal, que poderia se distanciar diante desse contexto. No casal 01 , Eduardo disse não saber o que aconteceria ao relacionamento se o tratamento não fosse bem sucedido, afirmando, ainda, que eles passaram por "uma fase de mais amizade do que de casamento”. No casal 02 , embora não se referisse espe- cificamente à sua própria relação, Sílvia comentou que, em casamentos em que "a coisa não estiver afinada”, a separação é, de fato, uma possibilidade. No casal 03, Viviana afirmava ao marido que, caso o tratamento não tivesse sucesso, eles iriam se separar. Esses comentários podem ser associados às constatações de autores que destacaram a influência negativa que a experiência da infertilidade pode exercer sobre as relações conjugais (Schaffer \& Diamond, 1994).

\section{Coesão: Durante a gravidez}

Para a esposa do casal 01 e ambos os cônjuges dos casais 02 e 03 , a gravidez foi vista como um elemento de união e fortalecimento da relação do casal. Segundo Daniel (casal 03 ), ele se sente "realizado de saber que nós tava $0 \%$ e agora nós estamos $100 \%$."

Outra questão identificada, nos relatos dos três casais, foi a valorização do companheirismo e dos diferentes tipos de apoio oferecidos pelo marido durante a gestação. De acordo com Sílvia, o marido "tem sido o que eu esperava que ele fosse: preocupado comigo, cuidadoso comigo, parceiro. Não me sinto sozinha nisso". Nos casais 02 e 03, também foram relatadas atitudes de cuidado e apoio das esposas em relação a seus maridos. Essas falas demonstram a percepção de uma proximidade emocional nesses casais, ou seja, que eles se sentiam coesos nesse momento. Diferentes estudos constataram que, em geral, a gravidez e a transição para a parentalidade tendem a ser marcadas por essa percepção de maior apoio (Piccinini, Gomes, Nardi, \& Lopes, 2008; Piccinini, Silva, Gonçalves, Lopes, \& Tudge, 2004). No entanto, é possível questionar o quanto as preocupações vivenciadas por esses casais durante a gestação - as quais podem ser relacionada a todo o período de dificuldades e incertezas vivenciado durante o tratamento - podem favorecer essas manifestações de cuidado. Fisher et al. (2008) constataram que mulheres que engravidaram com o auxílio das TRA apresentaram escores mais elevados do que a média das gestantes na dimensão cuidado, que avalia o afeto, o companheirismo, a confiança e a sensibilidade presentes no seu relacionamento com o marido.

Por outro lado, segundo os casais 01 e 02, com a gravidez, as esposas passaram a demandar mais a proximidade e o apoio de seus maridos, o que, de acordo com Ricardo (casal 02), é algo que "envaidece, mas também sobrecarrega". Conforme visto anteriormente, embora se tenha constatado que gestantes que conceberam com o auxílio das TRA tenderam a melhor avaliar o cuidado presente em seus relacionamentos (Fisher et al., 2008), Cohen et al. (2000) verificaram que homens cujas esposas engravidaram com o auxílio dessas técnicas avaliaram essa mesma dimensão de forma mais negativa do que o grupo controle. Dessa forma é possível supor que os cuidados prestados à esposa lhes gerem também certa sobrecarga, tanto física como emocional (Hackel \& Ruble, 1992).

Constataram-se, portanto, mudanças na dimensão coesão tanto em função do tratamento como da própria gestação. Embora o tratamento possa se mostrar uma oportunidade para o casal se unir em torno de um objetivo comum e tenha-se destacado o companheirismo dos maridos nesse momento, observou-se que esse período tende a apresentar um desafio 
em termos da continuidade do vínculo conjugal, visto que a relação do casal, em si, pode ser deixada de lado em prol de uma meta maior: a conquista da gravidez. Já no que se refere à gestação, o companheirismo e a união foram aspectos ressaltados, o que pode estar relacionado à própria experiência do tratamento. Deve-se considerar ainda que se trata de uma gravidez muito desejada por esses casais e em nome da qual os mesmos fizeram diversos sacrifícios pessoais e financeiros. Dessa forma, é compreensível que haja um forte interesse e preocupação em preservá-la. O participante que relatou um distanciamento durante a gestação (Eduardo - casal 01) foi justamente o que também demonstrou um maior afastamento durante a realização do tratamento. Este é considerado um momento mais estressante para o casal (Leiblum, Aviv e Hamer, 1998), o que demonstra que nem sempre as dificuldades vivenciadas nesse período são superadas com a gestação.

\section{Sexualidade: Durante o tratamento}

No que se refere ao exercício da sexualidade durante o período do tratamento, Eduardo (casal 01), Sílvia (casal 02) e Daniel (casal 03) mencionaram a necessidade de adequar sua vida sexual, corroborando o que é sugerido pela literatura (Leiblum, 1997). Sílvia revelou, inclusive, sua insatisfação a esse respeito, mencionando a perda da espontaneidade no casal. A literatura sugere que a experiência da infertilidade tende a exercer um impacto negativo sobre a sexualidade (Sabatelli et al., 1988). No entanto, de acordo com os relatos de Sílvia e Daniel, constatou-se que esse impacto pode não ser sentido imediatamente. Segundo ele, no princípio, houve até uma melhora na sexualidade, pois havia uma esperança, no casal, de conseguir engravidar sem a necessidade do tratamento. Observou-se, ainda, que esse impacto pode inclusive não ser identificado por algumas pessoas. Bianca (casal 01), por exemplo, relatou não ter percebido mudanças. Essa colocação, porém, pode ser analisada a partir da descrição da história do casal. Uma vez que ela mencionou que o sexo nunca foi um aspecto central em sua relação, é possível que algumas mudanças não tenham sido tão valorizadas.

\section{Sexualidade: Durante a gravidez}

Eduardo (casal 01) e Daniel (casal 03) destacaram que, com a gestação, houve uma diminuição em comportamentos tais como abraçar, namorar e "ficar agarrado". Essa diminuição é justificada, por eles, pelas mudanças corporais sofridas pela esposa e pelo aumento das atenções destinadas ao bebê.

Os três casais concordaram que houve, nesse período, um retraimento da sexualidade. Bianca (casal 01) não atribuiu essa mudança apenas à gravidez, mencionando a comodidade, a preguiça, a diminuição do desejo com o decorrer da relação e a insatisfação de ambos com seu próprio corpo. No entanto, demonstrou preocupação e dúvida a respeito do que essa diminuição poderia representar para seu marido, questionando se seria uma tentativa de cuidado ou um pretexto para se acomodar. Sílvia (casal 02), por outro lado, relatou que, no início da gravidez, o casal buscou outras formas de vivenciar sua sexualidade, mas, com o passar do tempo, isso também ficou impossível em função de suas mudanças corporais. Mais uma vez, pode-se pensar na importância de características anteriores da relação, visto que o casal 02 , em que a sexualidade era mencionada como um dos pontos fortes da relação, buscou alternativas que lhes permitissem continuar vivenciando-a.

Uma questão que pôde ser identificada no relato dos três maridos participantes deste estudo se refere ao medo de que o sexo pudesse prejudicar a gestação. Para Eduardo (casal 01) e Ricardo (casal 02), esse medo mostrou-se relacionado à realização do tratamento. Eduardo relacionou sua ansiedade de que algo pudesse acontecer ao bebê ao "defeito no esperma”, enquanto Ricardo afirmou: “O casal que já passou por isso e perdeu uma vez, é uma carga tão grande que, se disserem para ti 'olha você tem que pegar um copo d'água, botar em cima da televisão todo dia', tu é capaz de fazer”. Este resultado corrobora os achados do estudo de Ulrich et al. (2004). Ao compararem dois grupos de casais, um grupo que concebeu naturalmente e outro que concebeu através de técnicas de reprodução assistida, esses autores observaram que os casais do último grupo tenderam a se mostrar mais insatisfeitos sexualmente, chegando a interromper as relações por medo de prejudicar o bebê.

No entanto, diante das dificuldades encontradas por esses casais na vivência de sua sexualidade, é possível que outras formas de manifestar seu afeto passem a ser mais valorizadas. $\mathrm{O}$ cuidado do marido em relação à esposa foi destacado pelo casal 01 e por Sílvia (casal 02). Ricardo (casal 02), por sua vez, ressaltou o cuidado que a esposa demonstrava em relação a ele. Estar sempre junto, beijar (Viviana - casal 03), seguir o que o outro diz (Daniel - casal 03), lembrar de datas especiais e sentir a falta do outro (Ricardo - casal 02) também foram atitudes mencionadas. Além disso, pôde-se observar que atitudes tais como acariciar e conversar com a barriga também constituíram um elemento de aproximação do casal, como afirmou Daniel (casal 03): "Principalmente no carinho ela me pede bastante. (...) Qualquer alisada na barriga ela já está muito feliz.(...) Para mim é, assim, o que ela me pede o nenê está pedindo. E ela me fala isso: não é eu que estou pedindo, é o nenê que está pedindo”. De acordo com Miller et al. (2003), demonstrações de afeto como essas permitem que o cônjuge observe a responsividade de seu parceiro, contribuindo, assim, para a satisfação conjugal.

As verbalizações dos casais demonstram que, de fato, as TRA podem trazer mudanças na sexualidade durante a época do tratamento e mesmo após a gravidez. Embora modificações na sexualidade sejam esperadas durante a gestação (Piccinini et al., 2008), questiona-se se a experiência do tratamento intensificou esse processo, visto que o receio de prejudicar o bebê pode ter sido incrementado por essa experiência, como sugerem as falas de Eduardo (Casal 01) e Ricardo (Casal 02). No entanto, os participantes deste estudo pareceram buscar outras formas de manifestar seu afeto, diante da dificuldade de expressá-lo fisicamente, o que se pôde perceber, inclusive, no cuidado entre os cônjuges. Obviamente deve-se considerar que dois casais deste estudo vivenciaram riscos adicionais durante a gestação, os quais podem ter contribuído para esse maior receio: gestação gemelar (casal 02) e ameaça de nascimento prematuro (casal 03). 


\section{Comunicação: Durante o tratamento}

Relataram-se algumas dificuldades de comunicação presentes na época do tratamento, no que se refere a compartilhar determinadas informações com o cônjuge (Sílvia - casal 02), a compreender os sentimentos do outro (Bianca - casal 01 e Ricardo - casal 02) e a expressar os próprios sentimentos (Eduardo - casal 01). Tais dificuldades puderam ser observadas com maior clareza no relato do casal 01. Segundo Bianca, o tratamento não foi um assunto sobre o qual conversaram muito. Embora não considere que isso lhes tenha feito falta, ela relatou que só percebeu o impacto que o tratamento estava exercendo sobre o marido quando sua mãe lhe chamou a atenção. Ele, por sua vez, mencionou: "Foi um prato cheio pra eu me controlar um pouquinho mais, não mostrar a emoção antes. Deixar ela botar pra fora as emoções, como ela sempre foi, e, pra mim, segurar”. Segundo Walsh (2006), homens que não conseguem expressar seus sentimentos, em momentos de crise, tendem a se distanciar emocionalmente de suas parceiras, sendo que uma forma de lidar com a tensão existente seria concentrar as atenções nas atividades profissionais. De acordo com o relato de Eduardo, foi isso o que aconteceu na relação do casal. É possível, portanto, que as dificuldades em expressar os próprios sentimentos tenham contribuído para o impacto, percebido por ele, do tratamento sobre a relação conjugal.

Os casais 02 e 03 destacaram, ainda, conflitos que vivenciaram durante esse período. No casal 02 , Ricardo conta que ele e a esposa tiveram discussões sérias a respeito da continuidade do tratamento caso a gravidez não ocorresse naquela tentativa. Após algum tempo, porém, decidiram que só voltariam a falar sobre isso quando, de fato, soubessem o resultado. No casal 03, foram relatadas muitas brigas durante esse período: "E ela sempre insistia e falava como me dando um ultimato 'ou a gente faz esse filho ou terminou'. Isso, no caso, era por causa do dinheiro, de ter o dinheiro, 'ou tu dá um jeito ou vamos parar por aqui"'. Em situações de crise, a ocorrência de conflitos é esperada, pois, nesses momentos, a capacidade de lidar com as próprias emoções e de se comunicar fica prejudicada (Walsh, 2006). No entanto, no caso de Viviana (casal 03), percebe-se que as ameaças de pôr fim ao relacionamento já eram um comportamento presente anteriormente, visto que Daniel comentou que, quando brigavam, no início da relação, ela chegava a ir embora e ele precisava buscá-la. Esses conflitos manifestam, portanto, uma forma de o casal se comunicar frente às dificuldades vivenciadas, percebendo-se a influência de características prévias da relação na forma como o casal se comunicou durante o tratamento.

\section{Comunicação: Durante a gravidez}

Diferentes autores (Bradt, 1995; Snyder et al., 2006) referem que a chegada de um filho tende a afetar a comunicação do casal. A partir dos relatos dos participantes deste estudo, foi possível constatar que esse processo de mudança tem início já na gravidez. Algumas semelhanças quanto a esse aspecto foram observadas entre os casais.
A ênfase dada ao bebê nas conversas foi um aspecto que se mostrou presente na fala dos três casais. Inclusive, para Eduardo (casal 01), o fato de o único assunto do casal ser a filha pareceu lhe gerar certo incômodo. Em alguns casos, no entanto, esse predomínio das conversas sobre os bebês não se mostrou presente desde o início da gestação. Ricardo (casal 02) relatou que apenas ao final da gravidez passaram a conversar sobre os filhos. Tal afirmação pode ser relacionada ao medo que ele referiu ter sentido de investir emocionalmente nos bebês, em função do receio de que a gravidez não seguisse até o fim.

Melhoras na expressão e na compreensão dos sentimentos um do outro e a redução das brigas também foram aspectos mencionados pelos casais 02 e 03 . Ricardo (casal 02) afirmou, por exemplo, que, com a gravidez, Sílvia passou a compreender melhor algumas de suas atitudes: "o homem tem muito essa visão, não sei da onde é que veio, do abastecedor, do provedor e eu sempre tive e agora muito mais forte do que antes, e ela, de algum modo, tá entendendo um pouco”. Os relatos do Casal 03 também evidenciam bem essas questões, sendo que se percebeu uma diminuição das brigas do casal, que eram bastante presentes durante o período do tratamento, assim como a ideia de que um deve buscar fazer o que o outro diz. Daniel, por exemplo, referiu: "se eu falo uma coisa, ela obedece, se ela fala, eu obedeço. Nossa, antes nunca isso acontecia. Um queria mandar mais que o outro Agora não. Agora é o que ela fala eu faço. O que eu falo ela faz". No entanto, deve-se estar atento a que, de acordo com Walsh (2006), a pressão pelo consenso familiar pode prejudicar a comunicação e a capacidade de resolução de problemas. Segundo essa autora, a qualidade de um relacionamento não deve ser avaliada pela ausência de conflitos, mas pela forma como são manejados, visto que a evitação do conflito, quando se prolonga, pode afetar o funcionamento familiar.

Puderam ser observadas ainda relações entre a comunicação e outra dimensão da relação conjugal: a coesão. No casal 03, as falas de Daniel demonstram que o fato de ele perceber o casal mais unido contribuiu para a comunicação do casal: "Antes, se eu tivesse com um problema, eu ficava mais para mim, porque eu sabia que falar para ela não adiantava. Agora não. Agora eu falo o problema que eu tenho pra ela e ela ajuda. A mesma coisa ela para mim". Por outro lado, no casal 01, a dificuldade de expor certos sentimentos, manifestada por Eduardo, que disse que “engole” certas coisas, parece estar relacionada ao distanciamento percebido por ele na relação conjugal, conforme foi observado já no período do tratamento.

Os relatos dos participantes permitem inferir, portanto, que a comunicação é mais um fator que pode estar relacionado à forma como os casais vivenciam a experiência das TRA, visto que pode estar relacionada à percepção de outras dimensões da relação conjugal (casais 02 e 03), assim como representa uma estratégia para lidar com as diversas mudanças e dificuldades que estão sendo vivenciadas por esses casais diante do tratamento e da chegada do bebê. Olson (1991) sugeriu o papel facilitador desempenhado pela comunicação, que, segundo ele, seria essencial para a coesão e a adaptabilidade dos casais. Nesse contexto, a não expressão dos sentimentos tenderia a contribuir para o 
distanciamento do casal e para uma sensação de sobrecarga e isolamento, conforme já fora sugerido por Walsh (2006). Tal constatação corrobora os achados de outros autores (Pasch, Dunkel-Schetter, \& Christensen, 2002), que sugeriram que a comunicação tende a mediar o impacto da infertilidade sobre a relação conjugal. Pode-se observar, ainda, que as demandas do tratamento, de fato, provocaram alguns conflitos nos casais (Sabatelli et al., 1988). No entanto, a forma como eles foram solucionados parece refletir características anteriores da relação.

\section{Considerações Finais}

Este estudo teve como objetivo investigar a relação conjugal durante o tratamento e a gravidez, em casais que engravidaram com o auxílio das TRA. Abordaram-se as mudanças ocorridas nesses momentos em três dimensões da relação: coesão, sexualidade e comunicação.

$\mathrm{O}$ tratamento mostrou-se fonte potencial de dificuldades para a comunicação, a sexualidade e a coesão. O receio de expressar os próprios sentimentos, a não compreensão dos sentimentos do outro, a restrição à vida sexual e a potencial ameaça que o fracasso do tratamento pode representar à continuidade da relação foram algumas das questões identificadas durante este período, embora se tenha destacado o companheirismo existente entre os cônjuges. Por outro lado, na gravidez, embora algumas dificuldades tenham permanecido, especialmente na sexualidade, em função do medo de perder o bebê, foi ressaltado pelos casais um aumento na coesão, assim como alterações na comunicação, principalmente a redução de conflitos e a ênfase dada ao bebê nas conversas do casal.

Identificou-se a interação das três dimensões avaliadas, de forma que foi possível observar que uma dimensão pode tanto compensar dificuldades vivenciadas nas demais como contribuir para o funcionamento destas. Os resultados obtidos neste trabalho permitiram constatar, por exemplo, que a coesão pode ajudar a superar as dificuldades que o casal vivencia em sua sexualidade durante a realização do tratamento e a gravidez, assim como pode favorecer uma melhor comunicação a partir do sentimento de que se pode contar com o outro e de que, portanto, é válido compartilhar os sentimentos com ele.

Este trabalho evidencia a importância de considerar diferentes dimensões da relação conjugal para a compreensão da experiência desses casais. Essa abordagem multidimensional (Mossman, Wagner, \& Feres-Carneiro, 2006) favorece a identificação dos diferentes aspectos que compõem esse fenômeno e a própria elaboração de estratégias de intervenção junto a essa população, que podem abordar tais dimensões como recursos a serem aproveitados e desenvolvidos pelos casais.

Algumas considerações devem ser realizadas quanto ao potencial do método selecionado para este estudo. Acredita-se que a escolha de um delineamento de estudo de caso coletivo e de uma análise qualitativa dos resultados favoreceu a contextualização dos dados obtidos (Robson, 1993) e a descrição da forma como a relação é vivenciada durante a experiência das TRA e a gravidez, segundo a visão dos próprios participantes. Essa abordagem mais descritiva pareceu favorecer a identificação dos diferentes aspectos que compõem esse fenômeno, permitindo corroborar a multidimensionalidade (Mossman et al., 2006) da relação conjugal.

A escolha de casos heterogêneos (Patton, 2002) também se mostrou adequada ao propósito deste trabalho. Apesar de todas as diferenças existentes entre os casais, foi possível identificar algumas semelhanças que podem auxiliar a prática dos profissionais que trabalham nesse contexto e que lidam com a diversidade dessa população.

A realização de um estudo transversal pode ser considerada, a princípio, como uma limitação deste estudo, visto que não foi possível acompanhar se, de fato, as expectativas desses casais se concretizaram. No entanto, dessa forma, foi possível aprofundar questões específicas à gravidez, um período de grande relevância e que, muitas vezes, não é destacado ao se falar sobre o ciclo de vida familiar.

Algumas limitações deste estudo também devem ser consideradas ao analisar os resultados obtidos. Uma delas se refere ao fato de que as vivências referentes ao período do tratamento foram acessadas apenas de forma retrospectiva. Assim, é possível que o tempo transcorrido e a própria vivência da gravidez tenham influenciado a forma como esse período foi avaliado. A experiência das TRA faz parte do passado desses casais. No momento da entrevista, eles vivenciavam a realização de uma meta: a concretização da gravidez e a proximidade do nascimento do bebê. É provável, portanto, que o relato desses casais a respeito da experiência das TRA seja diferente daqueles que estão passando pelo tratamento ou daqueles que não conseguiram alcançar a gravidez.

Embora se tenha buscado certa variedade nos casos selecionados, é necessário destacar que a população alvo deste estudo é bastante específica: casais que conseguiram engravidar e que se mantiveram juntos ao longo do processo. De acordo com Sydsjö et al. (2002), trata-se de casais que encontraram formas de impedir que o estresse vivenciado pusesse fim à sua relação. Casais que se separam em função desse processo provavelmente descreverão de maneira diversa sua coesão, sua sexualidade e sua comunicação durante o tratamento. Além disso, os casais participantes deste estudo realizaram apenas uma ou duas tentativas para engravidar através das TRA. Segundo alguns autores (Repokari et al. 2007), o número de tentativas realizadas pelo casal tende a afetar sua adaptação a essa experiência. Dessa forma, seria interessante que fossem desenvolvidos estudos que acompanhassem os casais desde o início da realização do tratamento, verificando as diferenças entre aqueles que engravidam e os que não engravidam, entre os que se separam e os que permanecem juntos e entre aqueles que se submeteram a diferentes números de tentativas.

Além disso, não fez parte dos objetivos deste estudo avaliar as diferenças entre a experiência da gravidez em casais que engravidaram com o auxílio das TRA e casais que conceberam naturalmente. Futuramente, podem ser desenvolvidos estudos que tracem um paralelo entre essas populações, a partir de uma abordagem qualitativa. 


\section{Referências}

Andolfi, M., Angelo, C., Menghi, P., \& Nicolo Corigliano, A. M. (1989). Por trás da máscara familiar: Um novo enfoque em terapia da família. Porto Alegre: Artes Médicas.

Berthoud, C. M. E. (2002). Visitando a fase de aquisição. In C. M. O. Cerveny, \& C. M. E. Berthoud (Eds.), Visitando a família ao longo do ciclo vital (pp. 29-57). São Paulo: Casa do Psicólogo.

Boss, P. G. (1980). Normative family stress: Family boundary changes across the life-span. Family Relations, 29, 445-450.

Bradt, J. O. (1995). Tornando-se pais: Famílias com filhos pequenos. In B. Carter \& M. McGoldrick (Eds.), As mudanças no ciclo de vida familiar: Uma estrutura para a terapia familiar (pp. 206-222). Porto Alegre: Artes Médicas.

Brazelton. T. B., \& Cramer, B. G. (1992). As primeiras relações. São Paulo: Martins Fontes.

Burns, L. H. (1987). Infertility as boundary ambiguity: One theoretical perspective. Family Process, 26, 359-372.

Carter, B., \& McGoldrick, M. (1995). As mudanças no ciclo de vida familiar: Uma estrutura para a terapia familiar. In B. Carter, \& M. McGoldrick (Eds.), As mudanças no ciclo de vida familiar: Uma estrutura para a terapia familiar (pp. 07-29). Porto Alegre: Artes Médicas.

Cerveny, C. M. O. (2002). Pensando a família sistemicamente. In C. M. O. Cerveny, \& C. M. E. Berthoud (Eds.), Visitando a família ao longo do ciclo vital (pp. 17-27). São Paulo: Casa do Psicólogo.

Cohen, J., McMahon, C., Tennant, C., Saunders, D., \& Leslie, G., (2000). Psychosocial outcomes for fathers after IVF conception: A controlled prospective investigation from pregnancy to four months post-partum. Reproductive Technologies, 10, 126-130.

Conselho Nacional de Saúde. (1996). Resolução n 196, de 10 de outubro de 1996. Retrieved from http://www.datasus.gov.br/ conselho/resol96/RES19696.htm.

Fisher, J. R. W., Hammarberg, K., \& Baker, G. H. W. (2008). Antenatal mood and fetal attachment after assisted conception. Fertility and Sterility, 89, 1103-1112.

Glick, I. D., \& Kessler, D. R. (1980). Marital and family therapy. New York: Grune \& Stratton, Inc.

Hackel. L. S., \& Ruble, D. N. (1992). Changes in the marital relationship after the first baby is born: Predicting the impact of expectancy disconfirmation. Journal of Personality and Social Psychology, 62(6), 944-957.

Hjelmstedt, A, Widström, A. M., \& Collins, A. (2006). Psychological correlates of prenatal attachment in women who conceived after in vitro fertilization and women who conceived naturally. Birth, 33, 303-310.

Klock, S. C., \& Greenfeld, D. A. (2000). Psychological status of in vitro fertilization patients during pregnancy: A longitudinal study. Fertility and Sterility, 73, 1159-1164.

Lavee, Y., McCubbin, H. I., \& Olson, D. H. (1987). The effect of stressful life events and transitions on family functioning and well-being. Journal of Marriage and the Family, 49, 857-873.

Laville, C. \& Dione, J. (1999). A construção do saber: Manual de metodologia em ciências humanas. Porto Alegre: Artmed.

Lee, T. Y., Sun. G. H., \& Chao, S. C. (2001). The effect of an infertility diagnosis on the distress, marital and sexual satisfaction between husbands and wives in Taiwan. Human Reproduction, 16, 1762-1767.
Leiblum, S. R. (1997). Love, sex, and infertility: The impact of infertility on couples. In S. R. Leiblum (Ed.), Infertility: Psychological issues and counseling strategies (pp. 149-166). New York: John Willey \& Sons.

Leiblum, S. R., Aviv, A., \& Hamer, R. (1998). Life after infertility treatment: A long-term investigation of marital and sexual functioning. Humam Reproduction, 13, 3569-3574.

Lopes, R. C. S., \& Menezes, C. C. (2003). Entrevista individual com cada futuro cônjuge no último semestre antes do casamento. Manuscrito não publicado, Universidade Federal do Rio Grande do Sul, Porto Alegre.

Lopes, R. C. S., Piccinini, C. A., Dornelles, L. M. N., Silva, I. M., \& Passos, E. P. (2007). Transição para a parentalidade e a relação conjugal no contexto da reprodução assistida: Da gestação ao primeiro ano de vida do bebê. Manuscrito não publicado, Universidade Federal do Rio Grande do Sul, Porto Alegre.

Lopes, R. C. S., Silva, I. M., Piccinini, C. A., \& Dornelles, L. M. (2007). Entrevista sobre a Relação Conjugal na Gestação. Manuscrito não publicado, Universidade Federal do Rio Grande do Sul. Porto Alegre.

McDaniel, S. H., Herworth, J., \& Doherty, W. J. (1994). Terapia familiar médica: Um enfoque biopsicossocial às famílias com problemas de saúde. Porto Alegre: Artes Médicas.

Miller, P. J. E., Caughlin, J. E., \& Huston, T. L. (2003). Trait expressiveness and marital satisfaction: The role of idealization processes. Journal of Marriage and Family, 65, 978-995.

Mossman, C., Wagner, A., \& Féres-Carneiro, T. (2006). Qualidade conjugal: Mapeando conceitos. Paidéia, 16, 315-325.

Murray, H. A. (2005). TAT - Teste de Apercepção Temática. São Paulo: Casa do Psicólogo. (Trabalho original publicado em 1943)

Nichols, W. C., \& Pace-Nichols, M. A. (2000). Childless married couples. In W. C. Nichols, M. A. Pace-Nichols, D. S. Becvar, \& A. Y. Napier (Eds.), Family development and intervention (pp. 171-188). New York: John Willey \& Sons.

NUDIF - Núcleo de Infância e Família. (1998a). Entrevista sobre a Gestação e as Expectativas da Gestante. Manuscrito não publicado, Universidade Federal do Rio Grande do Sul, Porto Alegre.

NUDIF - Núcleo de Infância e Família. (1998b). Entrevista sobre a Gestação e as Expectativas do Futuro Pai. Manuscrito não publicado, Universidade Federal do Rio Grande do Sul, Porto Alegre.

Olson, D. H. (1991). Tipos de família, estrés familiar y satisfaccíon com la família: Uma perspectiva del desarrollo familiar. In C. Falicov (Ed.), Transiciones de la família: Continuidad y cambio en el ciclo de vida (pp. 99-129). Buenos Aires: Amorrortu.

Olson, D. H. (2000). Circumplex model of marital and family systems. Journal of Family Therapy, 22, 144-167.

Pasch, L. A., Dunkel-Schetter, C., \& Christensen, A. (2002). Differences between husbands' and wives' approach to infertility affect marital communication and adjustment. Fertility and Sterility, 77(6), 1241-1247.

Patton, M. Q. (2002). Qualitative research \& evaluation methods. Thousand Oaks: Sage Publications.

Pereira, C. R. R., \& Piccinini, C. A. (2007). O impacto da gestação do segundo filho na dinâmica familiar. Estudos de Psicologia (Campinas), 24, 385-395. 
Piccinini, C. A., Gomes, A. G., Nardi, T. D., \& Lopes, R. S. (2008). Gestação e a constituição da maternidade. Psicologia em Estudo, 13(1), 63-72.

Piccinini, C. A., Silva, M. R., Gonçalves, T. R., Lopes, R. C. S., \& Tudge, J. (2004). O envolvimento paterno durante a gestação. Psicologia: Reflexão e Crítica, 17(3), 303-314.

Pittman, F. S. (1987). Turning points: Treating families in transition and crisis. New York: W. W. Norton \& Company.

Repokari, I., Punamäki, R. I., Unkila-Kallio, I., Vilska, S., Poikkeus, P. P., Sinkkonem, J., Almqvist, F., Tiitnem, A., \& Tulppala, A. (2007). Infertility treatment and marital relationships: A 1-year prospective study among successfully treated ART couples and their controls. Human Reproduction, 22(5), 1481-1491.

Rios-González, J. A. (2005). Los ciclos vitales de la família y de la pareja: Crises u oportunidades? Madrid: Editorial CCS.

Robson, C. (1993). Real world research: A resource for social sciences and practioner researcher. Oxford: Blackwell.

Sabatelli, R. M., Meth, R. L., \& Gavazzi, S. M. (1988). Factors mediating the adjustment to involuntary childlessness. Family Relations, 37, 338-343.

Schaffer, J. A., \& Diamond, R. (1994). Infertilidade: Dor pessoal e estigma secreto. In E. Imber-Black (Ed.), Os segredos na família e na terapia familiar (pp. 113-127). Porto Alegre: Artes Médicas.

Seger-Jacob, L. (2006). Estresse na gênese e no tratamento da infertilidade. In R. M. M. Melamed \& J. Quayle (Eds.), Psicologia em reprodução assistida: Experiencias brasileiras (pp. 121-153). São Paulo: Casa do Psicólogo.

Shapiro, C. H. (1982). The impact of infertility on the marital relationship. Social Casework, 63, 387-393.

Sharf, C. N., \& Weinshel, M. (2002). Infertilidade e gravidez tardia. In P. Papp (Ed.), Casais em perigo: Novas diretrizes para terapeutas (pp. 119-144). Porto Alegre: Artmed.

Silva, I. M., \& Lopes, R. C. S. (2009). Reprodução assistida e relação conjugal durante a gravidez e após o nascimento do bebê: Uma revisão da literatura. Estudos de Psicologia (UFRN), 14, 223-230.

Snyder, D. K., Cozzi, J. J., \& Mangrum, L. F. (2006). Conceptual issues in assessing couples and families. In H. A. Liddle, D. A. Santisteban, R. F. Levant \& J. H. Bray (Eds.), Family psychology: Science-based interventions (pp. 69-87). Washington: American Psychological Association.
Spotorno, P. M., Silva, I. M., \& Lopes, R. C. S. (2008). Expectativas e sentimentos de mulheres em situação de reprodução medicamente assistida. Aletheia, 28, 104-118.

Stake, R. E. (1995). The art of the case study research. Thousand Oaks: Sage Publications.

Stanton, F., \& Golombok, S. (1993). Maternal-fetal attachment during pregnancy following in vitro fertilization. Journal of Psychosomatic Obstetrics Gynaecology, 14, 153-158.

Stern, D. N. (1997). A constelação da maternidade: O panorama da psicoterapia pais/bebê. Porto Alegre: Artes Médicas.

Sydsjö, G, Wadsby, M., Kjellberg, S., \& Sydsjo, A. (2002). Relationships and parenthood in couples after assisted reproduction and in spontaneous primiparous couples: A prospective long-term follow-up study. Human Reproduction, $17,3342-3250$.

Tudge, J., \& Frizzo, G. F. (2002). Classificação baseada em Hollingshead do nível socioeconômico das famílias do estudo longitudinal de Porto Alegre: da Gestação à escola. Manuscrito não publicado, Universidade Federal do Rio Grande do Sul, Porto Alegre.

Ulrich, D., Gagel, D. E., Hemmerling, A., Pastor, V. S., \& Kentenich, H. (2004). Couples becoming parents: Something special after IVF? Journal of Psychosomatic Obstetrics \& Gynecology, $25,99-113$.

Walsh, F. (2006). Strengthening family resilience. New York: The Guilford Press.

Wright, L. K. (1998). Affection and sexuality in the presence of Alzheimer's disease: A longitudinal study. Sexuality and Disability, 16, 167-179. 